\title{
Universal compact lower limb turning module intended for use in orthotic robots
}

\author{
Mateusz Janowski ${ }^{1}$, Danuta Jasińska-Choromańska ${ }^{1}$, Dymitr Osiński ${ }^{1}$, Marcin Zaczyk ${ }^{1, *}$ \\ ${ }^{1}$ Faculty of Mechatronics, Warsaw University of Technology, Boboli 8, 02-525, Warsaw, Poland
}

\begin{abstract}
In this paper, a model of an orthotic robot's lower limb rotation system is presented. The system is intended for use in typical contemporary orthotic robots such as the 'Veni-Prometheus' System for Verticalization and Aiding Motion designed at the Faculty of Mechatronics, Warsaw University of Technology. In the paper, the state of the art is briefly stated, with the relatively low number of orthotic robots allowing realization of pivoting turns highlighted. The intended two-stage pivoting turning movement is analyzed in detail and the operating conditions as well as limitations of the turning module are indicated. The conception of a turning module introduces additional degree of freedom to the existing orthotic robot designs by realizing the rotation about the lengthwise axis in the thigh link. A three-dimensional model and its analysis are shown. The proposed design ensures the necessary movement of the lower limb and the torso of an impaired person during the execution of pivoting turn while remaining compact in order to ease the introduction of the turning system to different orthotic robot designs.
\end{abstract}

Keywords: lower limb turning module, orthotic robot, exoskeleton, change of the gait direction, spinal cord injury, rehabilitation.

\section{Introduction}

In the paper, a concept of a turning module of the lower limb of an orthotic robot is proposed. The module is designed to be installed in the existing mechanical structures of orthotic robots, called also exoskeletons for aided rehabilitation, or to be implemented in a completely new structure. The task of the module is to enhance capabilities of the devices it is intended to work with, by the way of introducing an additional degree of freedom of the lower limb, which enables a change of direction of motion in a way resembling the natural one. At present, design solutions enabling a turn of the lower limb occur only in a few industrial and military exoskeletons.

Orthotic robots provide a possibility of motion, while the user is supported on crutches or on a walking frame (as in the case of ReWalk [1] and Ekso GT [2] robots), as well as without any additional supports (as in the case of REX [3] robot). The way of motion is considerably limited. Most of devices of this type make it possible for the handicapped to move by themselves only over a plane surface. But a few enable sitting down and rising up, as well as going up/down the stairs. Such movements as a rotation around the vertical axis

* Corresponding author: $\underline{\text { m.zaczyk@mchtr.pw.edu.pl }}$

Reviewers: Ivan Kuric, Bogdan Posiadala 
or a change of direction of motion in orthotic robots without an additional turning module, are realized by the way of raising a considerable part of the body weight using crutches or a walking frame and performing a turn on the feet of the device.

At the Institute of Micromechanics and Photonics, Faculty of Mechatronics, Warsaw University of Technology, a 'Veni-Prometheus' System for Verticalization and Aiding Motion (Fig. 1) was built, which was designed to restore lost motor abilities for the handicapped as well as to aid rehabilitation of individuals, who were not fully able $[4,5]$. The device offers five functions: moving over a plane, sitting down and rising up as well as going up/down the stairs. Electric motors drive movements of flexion and extension of the hip and knee articulations. The movement of dorsal and plantar flexion at the ankle joint is not driven. Works aimed at development of a mechanical structure providing a capability of making turns are currently being carried out [6].

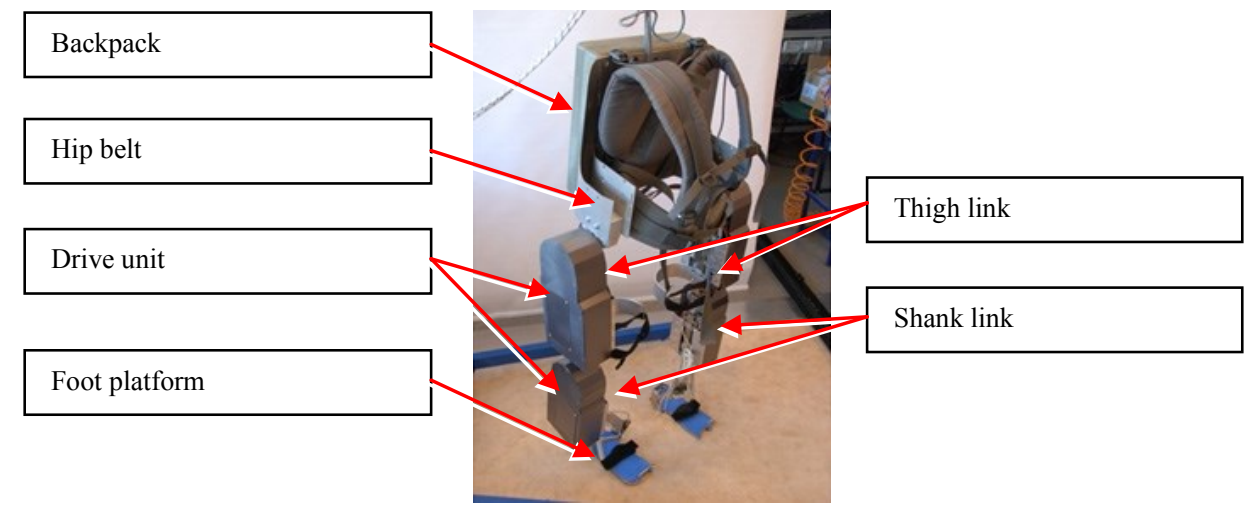

Fig. 1. 'Veni-Prometheus' System for Verticalization and Aiding Motion

\section{Survey of the existing solutions}

Orthotic robot equipped with a turning module is designed for individuals suffering from a serious injury of the spinal cord, such as accident victims with an injury of the lumbar region of the backbone that have completely or partially lost nervous connection between muscles and the brain from the waist down. Usually, they are not able to keep a vertical position using crutches or a walking frame due to an unsatisfactory stability of the lower limbs. A considerable part of such victims is confined to wheelchair. It is not an advantageous solution with regard to functioning of the organism, since it is limited by a sitting or lying position.

Wearable robots aiding movements of muscles, i.e. exoskeletons, which appear in civil industry as well as those created for military purposes, feature degrees of freedom that make it possible to change direction of motion. A common feature of almost all the solutions of this type is a lack of assistance for the degrees of freedom that are responsible for rotations enabling a change of direction of motion. Description of design solutions applied in outstanding exoskeletons (whose degrees of freedom of the lower limbs are not limited to the sagittal plane) are presented blow; these are NASA X1 (Fig. 2) and BLEEX (Fig. 3) exoskeletons.

The NASA X1 exoskeleton was designed for the purposes of dynamometric measurements as well as trainings for astronauts [7]. Because of micro-gravitation conditions in space bases and spacecrafts, muscles and bones of astronauts are subjected to a fast degeneration. At the initial stages, the design was also intended for individuals suffering from an injury of the spinal cord. Thus, forces resulting from the gravitation of the 
user's mass are transmitted through the frame of the exoskeleton to the ground. The assisted degrees of freedom are flexion and extension at the knee as well as flexion and extension of the thigh. The exoskeleton enables a turn of the leg, devoid of its drive, at the hip owing to application of a hinge located between the hip frame and the thigh section of the exoskeleton (Fig. 2).
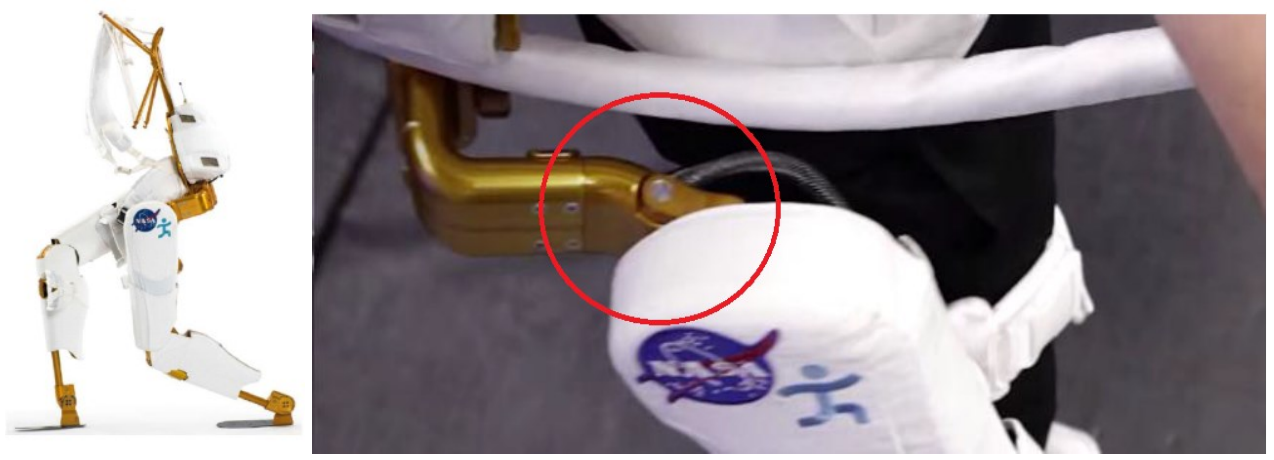

Fig. 2. NASA X1 and its turning module $[7,8]$

Another example is BLEEX exoskeleton, which was designed to help carry large masses that are secured in the backpack fixed to the frame of the device [9]. Forces resulting from the gravitation of the backpack mass are almost completely transmitted by the frame of the exoskeleton, in order to reduce fatigue of the user owing to smaller loading of his joints and muscles. The assisted movements are flexion and extension at hip joints, knee joints and ankle joints as well as adduction and abduction at hip joints. It is also possible to perform movements of metatarsus flexion and hip rotation, however the movements are not powered. The movement of rotation about the vertical axis is possible both at the articulations located above the thigh connectors as well as at the middle articulation connecting both halves of the hip belt, what made it possible for the authors of the design to compare gaits performed using one of the two options (Fig. 3).
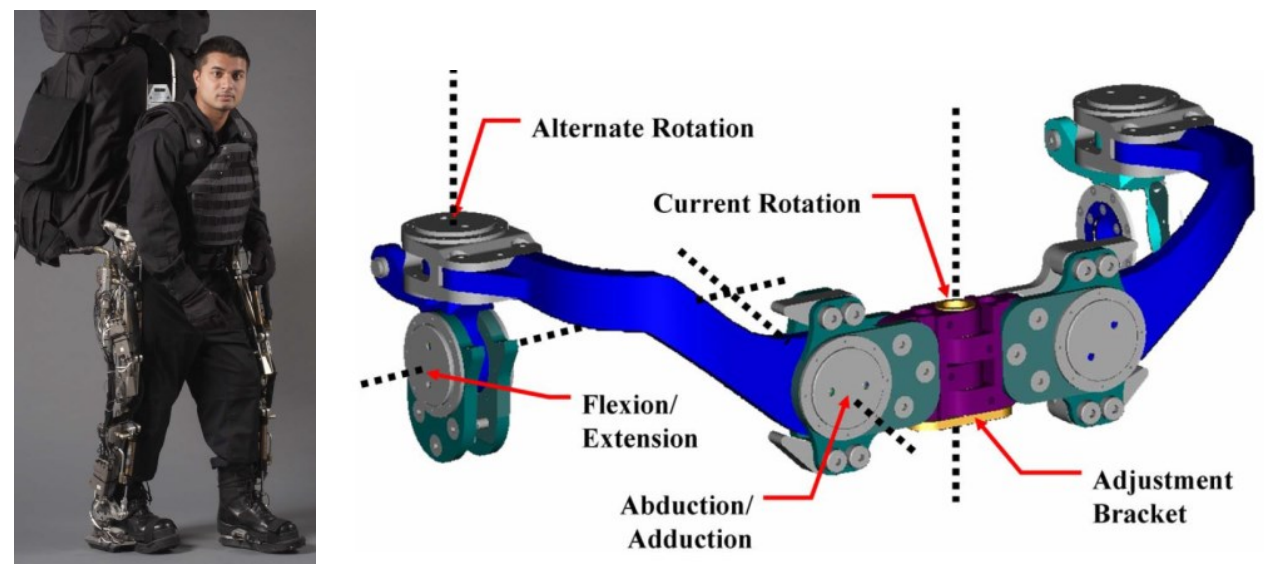

Fig. 3. Hip belt of BLEEX exoskeleton [9]

Both in the NASA X1 as well as in the BLEEX exoskeleton, rotation of the lower limb is possible owing to application of hinges enabling a motion within a transverse plane. In both designs, the rotation axis is shifted out of the outline of the human body. That results in linear displacements between the exoskeleton connector and the leg of the user while a 
rotation is performed, and the articulations connected with rotation of the lower limb within a transverse plane are devoid of a drive, what in turn results in the necessity of the user to be able to perform appropriate movements in order to initiate the turning by himself.

\section{Conception of the lower limb turning system in the orthotic robot}

In order to improve the way of locomotion provided by the existing orthotic devices that aid motion of the handicapped, one may apply an additional system making it possible to introduce a driven degree of freedom of the lower limbs, which in fact enables a turn of the lower limb at the hip with respect to the vertical axis, i.e. a hip rotation in order to ensure a possibility of changing direction of motion, i.e. turning.

It seems that the most advantageous solution to be implemented in the existing robots is a separate module of additional degree of freedom, making it possible to change direction of motion by the way of introducing a driven hip rotation. Such solution is aimed at an easy adaptation and assembly of the device in the existing orthotic robots without a necessity of introducing considerable changes of the mechanical structure.

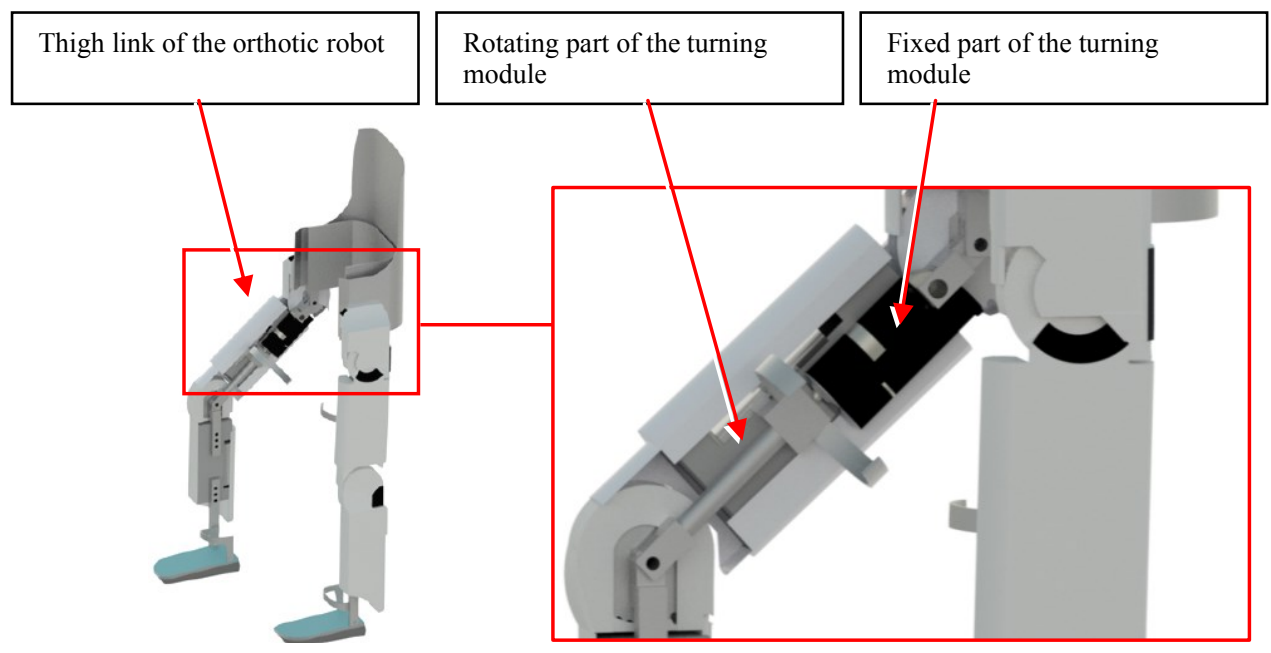

Fig. 4. Practical realization of the foot turning module in the Veni-Prometheus orthotic robot with close-up view of the applied turning module of the lower limb

While designing the turning module, one chose a solution of turning the lower limb, which consists in rotation about an axis that is parallel to the thigh within a transverse plane. It was decided to accept such solution also due to a possibility of obtaining smaller overall dimensions and masses as compared to other solutions. 


\section{Results}

\subsection{Methodology for determination of the operating conditions for a hip rotation system}

The analysis covered two basic cases of loads during operation of the considered foot turning module. It was assumed that a turn of the lower limb will be realized in four stages. At the first stage, one of the lower limbs will be raised by the way of a flexion at the hip and the knee, thus it will turn outwards. At the second stage, a turn of the trunk will take place in such a way that the body, except for the leg resting upon the ground, is directed toward direction of the turn. At the third stage, the raised leg will be lowered to the ground, and at the fourth stage the leg that has been resting on the ground so far, will be raised, turned and set next to the second one.

For such scheme, one can distinguish two main cases of loads of the turning mechanism, i.e. when:

- the limb, upon which the turn is realized, is the main point of support of the exoskeleton,

- the limb is turned at the moment of being raised above the ground to a height of $50 \mathrm{~mm}$, thus ensuring a collisionless turn of the foot.

The first indicated case assumes a rotation of the trunk with respect to the motionless lower limb supported on the ground. User of the orthotic robot makes use of crutches or a walking frame in order to have a support, what results in the fact that he has an additional support and can perform a rotation of the trunk using the upper groups of the muscles. Owing to such approach, the motion can be more fluent and predictable for the user, since he controls it himself, what may result in a better balance. That makes it also possible to use a smaller drive since there is no need to force and brake a motion of almost the whole body; only the lower limb is to be driven, in the second case.

The second case assumes a rotation of the lower limb, when it is raised by an appropriately small angle at the hip. It was assumed that before rotation of the limb, the shank will be positioned orthogonally with respect to the ground. Additionally, the feet of the device are always parallel to the shank, what results from the design of typical orthotic robots.

In order to calculate value of the forces and the torques occurring in the above cases, the following main assumptions were accepted:

- maximal permissible mass of the user is $110 \mathrm{~kg}$,

- user's height is $1.8 \mathrm{~m}$,

- Veni-Prometheus is considered as the exoskeleton the turning module is mounted to and has mass of $20 \mathrm{~kg}$,

- the lower limb of the exoskeleton/orthotic robot has mass of $5 \mathrm{~kg}$,

- turning module of the lower limb is fixed to one of the lower limbs and has mass of 1.5 $\mathrm{kg}$,

- turning module of the lower limb is not rotated with the limb, onto which it is attached,

- time of rotating the limb while it is lifted above the ground is $0.5 \mathrm{~s}$.

The considered case concerns loads of the drive while rotating a lifted lower limb, since such situation defines requirements related to the operation of the mechanism the best way. It is a conservative assumption, as it is the case with the largest loads in the turning mechanism. 


\subsection{Computation of the mechanism's load}

\subsubsection{Rotation of lifted lower limb}

The first issue taken into consideration was to define the angle by which the lower limb will be lifted with respect to the trunk. In Fig. 6, this angle is denoted as $\alpha$. The other denotations mean:

$L$ - length of thigh/shin together with the foot,

$R$ - distance between the foot and the rotation axis of the lower limb,

$d x$ - distance between the sole and the ground,

$\alpha$ - angle at which the thigh is lifted with respect to the trunk.

It was assumed that the minimal distance $\mathrm{dx}$ should be $50 \mathrm{~mm}$ in order to ensure that rotation of the limb will not result in a collision, and thus the angle at which the thigh was lifted was evaluated at $26^{\circ}$.

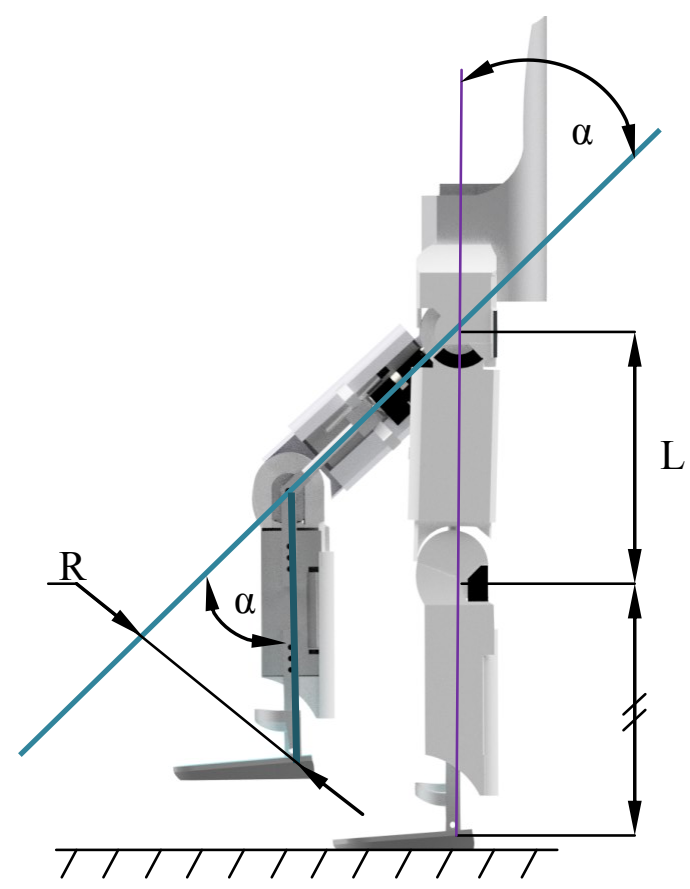

Fig. 5. Geometry of the computed arrangement

\subsubsection{Consideration related to mass moments of inertia}

The rotation axis of the lower limb is an axis parallel to the long thigh part of the lower limb. During a motion, the shin is inclined by an angle $\alpha=26^{\circ}$ with respect to the rotation axis. In order to determine the mass moment of inertia of the limb while rotated around the considered axis, the following were taken into account:

- displacement between the mechanism (thigh connector) and the user's body is $150 \mathrm{~mm}$,

- simplification that the user's body density is homogeneous,

- mass moments of inertia of the user's body resulting from its model,

- mass moments of inertia of the mechanism,

- increase of inertia due to shift of the axis, calculated according to the parallel axis theorem. 
While computing the mass moment of inertia, a parametric model of the human body made in the MSC Adams software was employed [10]. The computations were executed for a possibly disadvantageous case of loads, when the user of the device is of the maximal weight and height. Size and mass of the turning module were over-evaluated in order to take into account additional resistance to motion, which results among other things from movements of the human body or a necessity of overcoming frictional resistance at the joints and articulations of the device.

\subsubsection{Calculation of angular acceleration and maximal rotational speed}

The assumed time of performing rotation of the limb at the hip is $0.3 \mathrm{~s}$ (shorter than the accepted requirement), and the angular way of that motion is $30^{\circ}$. Because of a relatively small range of operation it has been assumed that the speed profile of the considered motion is triangular, what means that within the first half of the angular way a constant acceleration is developed, whereas a constant deceleration within the second half. Thus, the angular acceleration was obtained, accepting the maximal time of motion and the required angular range of the rotation.

The considered case is related to loads of the drive while rotating a lifted lower limb, being the most representative with regard to the requirements connected with operation of the mechanism, since at the assumed way of performing a turn the biggest loads of the turning module occur.

\subsubsection{Resultant maximal torque}

Knowing the maximal computed values of the mass moment of inertia as well as the angular acceleration, it was possible to determine a maximal value of the torque resulting from accelerating the inert mass of the lower limb. The value was calculated on the basis of the mass moment of inertia of the member as well as a constant angular acceleration of the motion. The final value was increased by ca. $10 \%$, taking into account a resistance to motion that has been neglected so far. Thus, the ultimate value was evaluated at $3.5 \mathrm{Nm}$.

\subsubsection{Required characteristics of the drive}

To drive the device, it was assumed that a drive unit will be used, which meets the following requirements:

- overall dimensions no larger than $150 \times 100 \times 100 \mathrm{~mm}$,

- supply voltage up to DC $24 \mathrm{~V}$,

- constant output torque of the drive unit exceeding $3.5 \mathrm{Nm}$,

- $\quad$ rotational speed of $20 \mathrm{rpm}(2.1 \mathrm{rad} / \mathrm{s})$ to be developed within $0.25 \mathrm{~s}$.

The requirements were specified on the basis of a survey of typical commercial electric DC micro-drives available.

A minimal power of the electric drive at the output drive was determined, taking into account the maximal required torque as well as the maximal rotational speed to be developed.

The computed value is only a part of the power output of the motor, and the power of the motor driving the mechanism should be higher both due to its efficiency as well as additional resistance to motion, which was not taken into account in the above considerations, such as friction between the mechanical members and resistance of a frictional character resulting from a necessity of setting in motion body parts of the user. Finally, it was assumed that the power output of the motor should be no lower than $20 \mathrm{~W}$, 
what is approximately 3 -times higher than the value computed above. Such a big difference results also from the fact that it is advantageous to use such part of the operational characteristic of the motor, where it features high efficiency, i.e. between $15 \%$ and $50 \%$ of the stall torque.

Brushed DC motors were surveyed. Due to typical characteristics of drives, the following were assumed:

- the motor works with a gear featuring reduction ratio over 100:1 and efficiency of at least $\eta=70 \%$,

- the required constant torque of the motor is approximately of $50 \mathrm{mNm}$,

while formulating the requirements, typical DC electric drives and reduction gears were considered. The required values calculated above pertain to the torque and the speed of the output shaft of the turning module, i.e. the element that drives directly rotation of the limb.

\subsubsection{Check of the output rotational speed}

Because of the required a torque having a large value at a low rotational speed it was decided to use an electric motor coupled with a reduction gearhead. The maximal speed at the output of the motoreducer should be no lower than the assumed 20 RPM. Knowing typical designs of drives that meet the specified requirements, a turning module of the feet was designed; it is presented in Fig. 6. Electric motor is coupled with a reducer transmits the drive to a rotary member coupled with an encoder by means of a belt transmission. Assembly of the unit (without the casing) is also presented in Fig. 6.

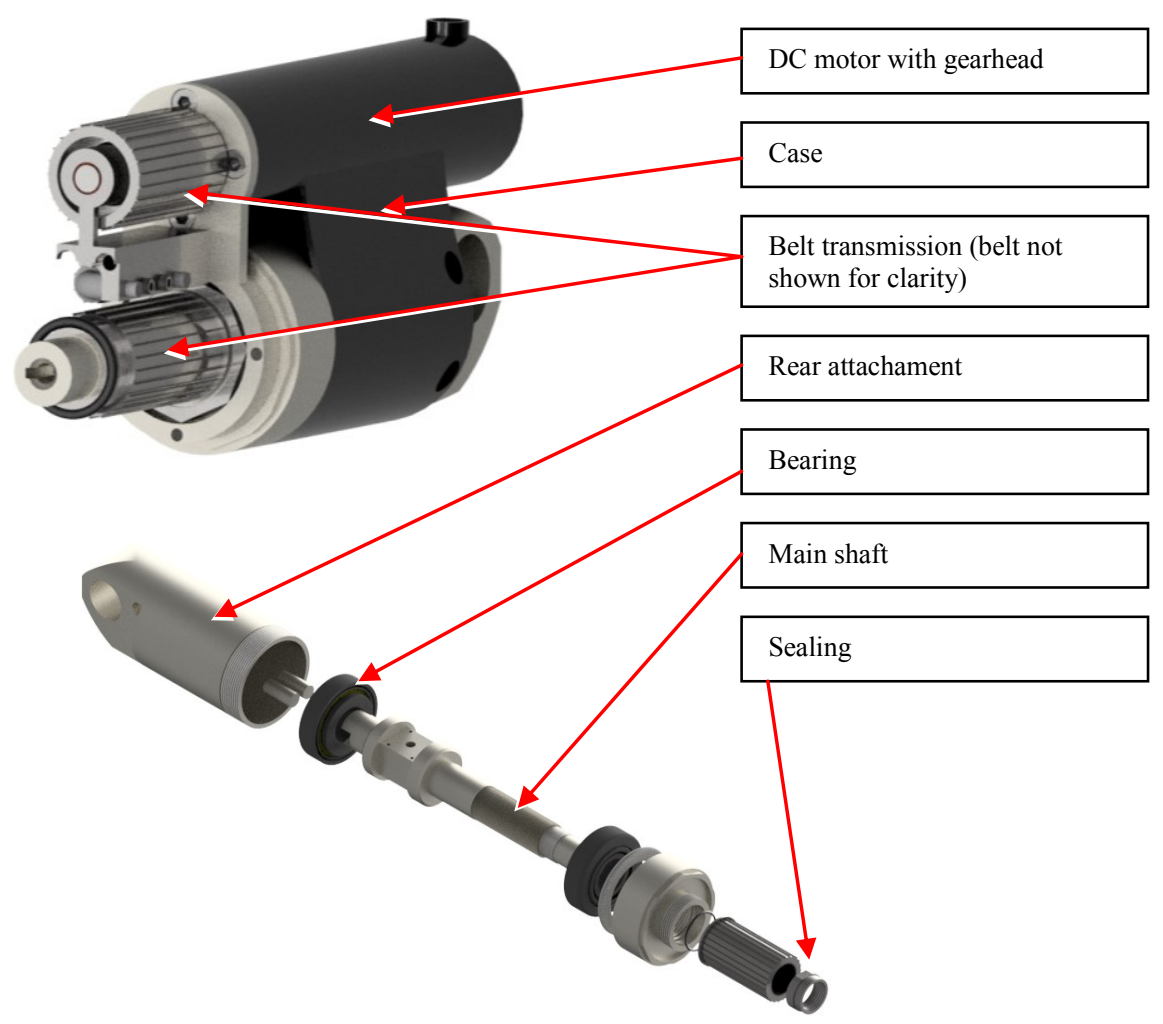

Fig. 6. Perspective and partially exploded view of the designed drive 


\section{Discussion}

The orthotic robot taken into consideration for operating with the presented module of foot turning is the 'Veni-Prometheus' System for Verticalization and Aiding Motion. Thanks to choosing the concept of the turning module that is a separate unit attached to the frame of the exoskeleton, providing additional driven degree of freedom of the lower limb, it is also possible to adapt the turning module for other orthotic robots.

The presented concept of the device is meant to enhance turning capabilities of orthotic robots for rehabilitation purposes as well as aiding motion of individuals suffering from a spinal cord injury. The way of performing movements while changing direction of motion is strictly defined. All the available degrees of freedom, which are featured by the orthotic robot working together with the additional module, are actuated, what makes it possible to ensure a motion range which is explicitly defined and is safe for the user. It is aimed at protection against a possible injury - pulling one's tendons or muscle attachments, performing movements that are not anatomical, or performing undesired movements of the articulations, which would not be driven otherwise.

Locating the turning module in the hip connector, which overlaps the exoskeleton's axis of the rotation movement, results however in some linear displacements during a rotation, since a human limb rotates around an axis that runs through the head of the thigh bone. Such displacement results in an increase of the load of the mechanism, both due to introduction of undesired displacements as well as an increase of the mass moments of inertia (according to the parallel axis theorem), which load the limb. However, in the case of such arrangement the mass moments of inertia connected with the exoskeleton are smaller, since the motion takes place just around its axis.

\section{Conclusions}

Among the currently used design solutions of orthotic robots, a turn of the lower limb in the hip was initially considered - just as in the case of NASA X1 and BLEEX exoskeletons. It was noticed that a turn of the leg may be realized along the thigh axis within a safe range of rotation of $30^{\circ}$. It was verified if the new device would realize a turn of the whole limb at any position with respect to the whole robot, and it was accepted that at the assumed parameters of motion, there always took place a correct turn of the lower limb.

Computations related to the drive for the new design solution were executed on the basis of the obtained results, and it was stated that the output power generated by the motor may be over three times lower as compared with the solution employing a turn in the hip. This results in lower mass and overall dimensions of the whole device, as well as lower consumption of electric energy while activating a turn of the lower limb.

\section{References}

1. A. Esquenazi, M. Talaty, A. Packel, M. Asulino, The ReWalk Powered Exoskeleton to Restore Ambulatory Function to Individuals with Thoracic-Level Motor-Complete Spinal Cord Injury. Am. J. Phys. Med. Rehabil. 91, 911-921 (2012)

2. T. Swift, Control and Trajectory Generation of a Wearable Mobility Exoskeleton for Spinal Cord Injury Patients. PHD Thesis, University of California, Berkeley (2011)

3. R. Irving, R. Little, Mobility aid. European Patent, EP 2231096 (2013)

4. D. Jasińska-Choromańska, K. Szykiedans, J. Wierciak, D. Kołodziej, M. Zaczyk, K. Bagiński, M. Bojarski, B. Kabziński, Mechatronic system for verticalization and 
aiding the motion of the disabled. Bulletin of the Polish Academy of Sciences, Technical Sciences 61 (2), 419-431 (2013)

5. K. Bagiński, J. Wierciak, Forming of operational characteristics of an orthotic robot by in-fluencing parameters of its drive systems. R. Szewczyk, C. Zieliński, M. Kaliczyńska, Adv. Intel. Sys. Comput. 351 (2), 1-9 (2015)

6. D. Osiński, M. Zaczyk, D. Jasińska-Choromańska, Conception of Turning Module for Orthotic Robot. R. Jabłoński, T. Brezina, Adv. Intel. Sys. Comput. 393, 147-152 (2016)

7. R. Rea, Ch. Beck, R. Rovekamp, M. Diftler, P. Neuhaus, X1: A Robotic Exoskeleton for In-Space Countermeasures and Dynamometry. AIAA Space 2013 Conference and Exposition (2013)

8. X1 - Exoskeleton for Resistive Exercise and Rehabilitation. [online] https://www.youtube.com/watch?v=XedjEBocaKo (2012)

9. A. Zoss, H. Kazerooni, A. Chu, On the Mechanical Design of the Berkeley Lower Extremity Exoskeleton (BLEEX). IEEE/RSJ, 3465-3472 (2005)

10. D. Osiński, D. Jasińska-Choromańska, Parametric Model of Human Body for Orthotic Robot Simulation Study. Adv. Intel. Sys. Comput. (to be published) 